\title{
“Double Bundle - Single Tunnel” Hamstring Graft Fixation in Femur
}

\section{Rajkovic Stanislav ${ }^{1 *}$, Vukomanovic Boris ${ }^{2}$ and Jeremic Danilo ${ }^{1}$}

${ }^{1}$ Departement for Orthopaedic, Institute for Orthopaedics "Banjica", Belgrade, Serbia

${ }^{2}$ Departement for Surgery, University of Belgrade, Serbia

*Corresponding Author: Rajkovic Stanislav, Departement for Orthopaedic, Institute for Orthopaedics “Banjica”, Belgrade, Serbia.
Received: October 31, 2020

Published: November 18, 2020

(C) All rights are reserved by Rajkovic

Stanislav., et al.

\section{Abstract}

Purpose: An original technique of introducing hamstring graft into the femoral tunnel and its' fixation which forms an anatomic double bundle anterior cruciate ligament (ACL).

Materials and Methods: Hamstring tendon graft is prepared with a "head", "body" and two "tails". The femoral tunnel is drilled at the Resident's and Bifurcation ridge junction through the anteromedial portal and subtunnels are prepared. The graft is introduced into the knee - "body" in the femoral tunnel, "tails" in one subtunnel and "head" in the other. Fixation is done by an absorbable interference screw. The "head" and the "tails" are twisted inside the tibial tunnel. Fixation in the tibial tunnel is done by an absorbable interference screw.

Discussion and Conclusion: This technique eliminates "bungee" and "windshield wiper" effect, anatomic position of the graft is achieved and risk of "blow out" effect and graft damage during screw insertion is significantly reduced.

Keywords: Cruciate; Ligament; Reconstruction; Arthroscopy; Sports; Injury

\section{Introduction}

Arthroscopic techniques of anterior cruciate ligament (ACL) brought to enormous increase in number of performed ACL reconstructions enabling increased stability and better function of the knee, shorter post-operative period and quicker recovery and comeback to regular every-day and sports activities [1]. It is estimated that one in 3000 persons would undergo ACL reconstruction [2]. Today two grafts are in everyday use [2, 3]: 1. patellar tendon (Bone-tendon-Bone, BTB), and 2. gracilis and semitendinosus muscle tendon (hamstring). Both are used at almost the same rate, both have some advantages and drawbacks and only surgeon's experience choses a better graft for a particular patient $[4,5]$. It is considered that ACL reconstruction procedure depends on achieving anatomical position and maintaining the graft in bone tunnels [3]. For the tibial tunnel most commonly an absorbable interference screw is used to rigidly fixate the graft [6]. Femoral tunnel graft fixation could be classified into two major groups: 1 . suspended fixation, and 2. rigid fixation. Suspended fixation means that the graft is attached to the tunnel only at its' top while through the rest of the tunnel it just leans on the bony walls [5]. Most commonly used techniques from this group are Endo button (ACL Tight Rope, Artrex) and transfemoral screw (Bio-TransFix, Artrex). Main drawbacks of this technique are the graft stretching - "bungee" effect, and circular or semi-circular movements of the graft inside the tunnel - "windmill" or "wind-shield wiper" effect, resulting in tunnel enlargement and graft loosening. This can affect graft ingrowth to the bone and bring to the procedure failure [7, 8]. Rigid fixation or as it is also known "near aperture" fixation is a method in which the graft is firmly positioned in the tunnel or at least at its' lower end (aperture) most commonly by an interference screw or by special transfemoral pins (RIGIDFIX Cross Pin System, DePuy Mitek). Drawbacks of this method are possible graft damage by the screw or posterior tunnel wall disruption - "blow out" effect [8]. 


\section{Material and Methods}

\section{Graft preparation}

First gracilis tendon is separated from the pes anserinus and $3 \mathrm{~cm}$ "fish bone" suture is made at distal end of the tendon using absorbable thread (we use PDS II, Polydioxanone Suture, Size 1, ETHICON) leaving some $10 \mathrm{~cm}$ of the thread free at the end of the graft. This end of the future graft is called the "tail". The tendon is then separated from the muscle and the same procedure is repeated at semitendinosus tendon. Beginning from "the tail" $20 \mathrm{~cm}$ is measured and the rest of the tendons is cut off. Tendons are placed parallel to each other and stitched together at the opposite end of the "tails" using the same "fish-bone" suture also leaving free some $10 \mathrm{~cm}$ of the thread. This end of the graft is called the "head". In this way a double graft, $20 \mathrm{~cm}$ long, with one "head" and two "tails" is obtained. The exact middle point of the graft is determined and using the same thread type and the same "fish-bone" technique another suture $6 \mathrm{~cm}$ long $(3 \mathrm{~cm}$ on each side from the midpoint of the graft) is made (this time we use softer thread VICRYL, Polyglactin 910 Suture, Size 2-0, ETHICON). This part of the graft is called the "body".

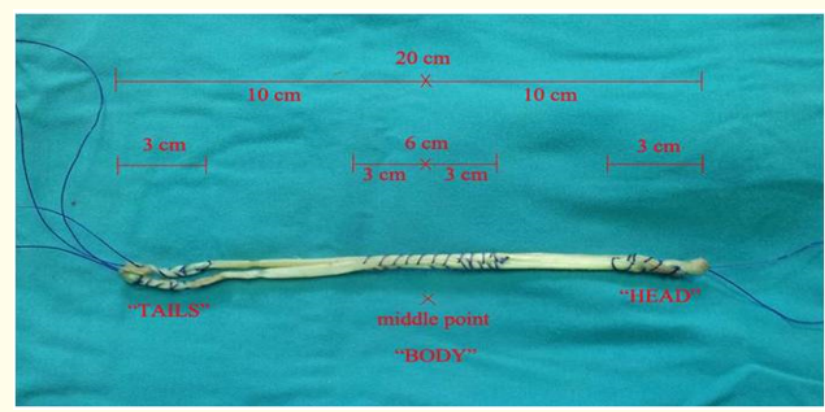

Figure 1: Graft.

\section{Tunnel preparation}

Knee is flexed to 110 - 120 degrees. A K-wire with en eyelet is introduced through the anteromedial portal to the knee and at the connection point of Resident's and Bifurcation Ridge is drilled until is exits the skin at lateral thigh. Along the K-wire drilling is made with a drill $0.5 \mathrm{~mm}$ wider than the width of the graft. A 3.5 $\mathrm{cm}$ long femoral tunnel is made. Then, using a $2-3 \mathrm{~mm}$ abrader, subtunnels are made. For the right knee subtunnels are positioned at 06:15 hours and for the left knee at 06:45 hours. Length of the subtunnels should be $10 \mathrm{~mm}$, width 3 - $4 \mathrm{~mm}$ and depth 2 - $3 \mathrm{~mm}$. Through the eyelet of the K-wire a guide thread around $30 \mathrm{~cm}$ long is pulled through and the wire is extracted on the lateral side of the knee pulling two ends of the thread with it. In this way a double thread with a loop going out form the anteromedial portal is obtained. Tibial tunnel is prepared the same way as at the other hamstring graft techniques.

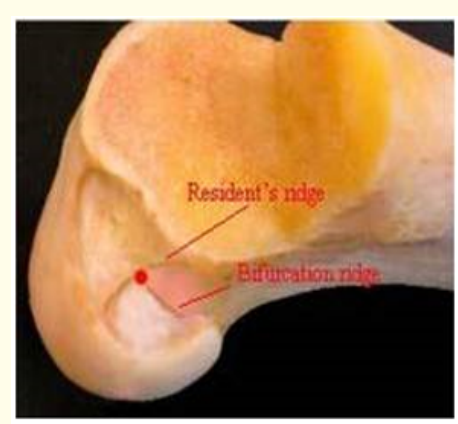

Figure 2: ACL footprint on femur.

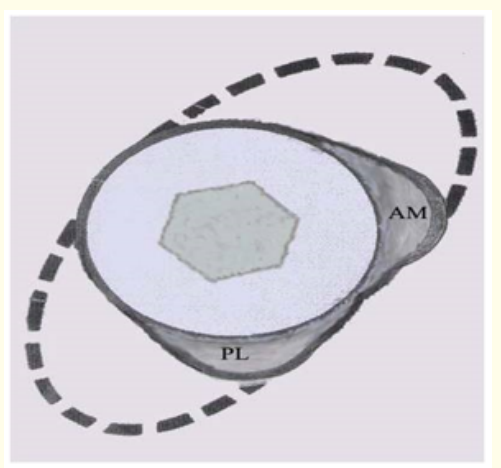

Figure 3: ALC footprint.

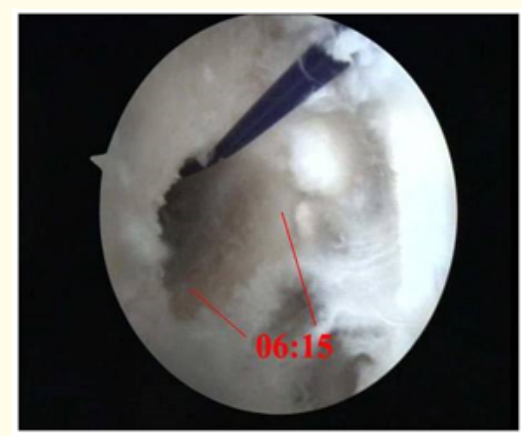

Figure 4: Femoral tunnel. 


\section{Graft introduction and fixation in the femoral tunnel}

Double graft is pulled through the loop of the guide thread. Through the anteromedial portal, one by one, the "tails" of the graft along with the free thread on its' end are introduced inside the knee and pulled out through the tibial tunnel using an instrument. The guide thread is pulled on the lateral side of the knee introducing the "body" of the graft into the femoral tunnel. In this way the "tails" of the graft are in the tibial tunnel, the "body" in the femoral tunnel and the "head" goes out of the knee through the anteromedial portal. An interference screw (we use MILAGRO, DePuy Mitek) is introduced into the femoral tunnel above the graft "body" ( 8 x $30 \mathrm{~mm}$ screw for 7.5 - $8 \mathrm{~mm}$ tunnel; 9 x $30 \mathrm{~mm}$ screw for 8.5 - $9 \mathrm{~mm}$ tunnel). During the screw insertion each double part of the graft must be laid down in one subtunnel - this is achieved by the separation of the graft ends - the "head" is in the anteromedial portal and it is placed in the 06:15 subtunnel for the right knee and 06:45 for the left; the "tails" are in the tibial tunnel and are placed in 06:15 subtunnel for the right knee and 06:45 subtunnel for the left.

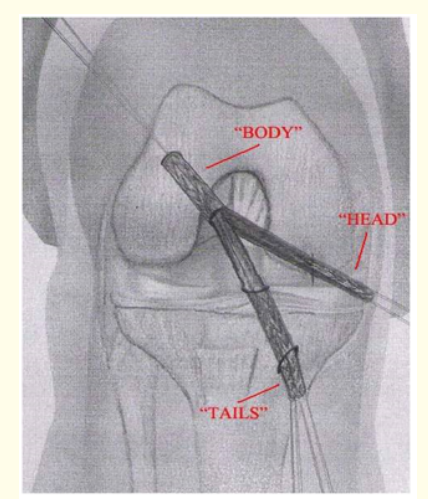

Figure 5: Graft introduction.

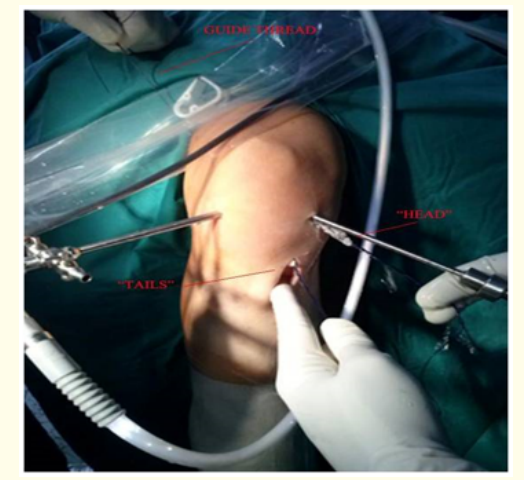

Figure 6: Graft position, operative view.

\section{Final positioning and graft fixation in the tibial tunnel}

In the same way as the two "tails" before, the "head" is inserted into the knee and pulled out through the tibial tunnel. Two "tails" held together and the "head" are twisted and positioned in the tibial tunnel so that final form of anatomic ACL is achieved and two major bundles are clearly visible. Fixation in the tibial tunnel is done in the same way as in other hamstring techniques using an interference screw, knee flexed at 30 degrees.

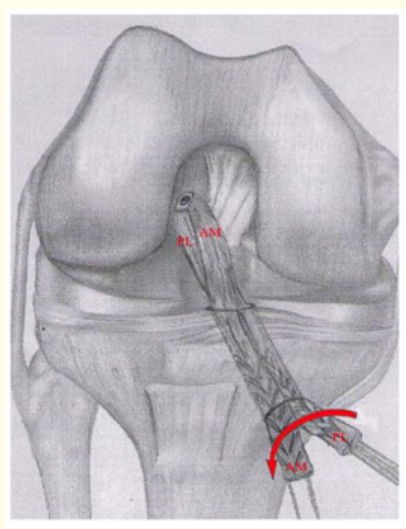

Figure 7: Final positioning.

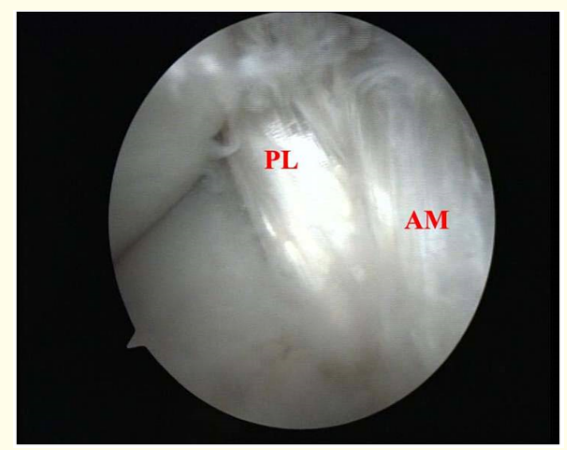

Figure 8: Reconstructed double bundle ACL.

\section{Discussion}

No specific ACL reconstruction technique satisfies all the needs of this procedure $[9,10]$. Today it is considered that the greatest influence on this surgical procedure success have two factors: 1 . anatomical position of the graft, and 2. fixation of the graft that can withstand long enough for the graft to grow into the bone and take form of natural ACL [11]. Along with these two factors special attention is directed to at least two major bundles of this ligament, anteromedial (AM) and posterolateral (PL) [12]. Surgical technique 
described above should be called "double bundle - single tunnel". Similar techniques had been described previously. Morgan CD., et al. [13] published a technique with femoral tunnel notches but technique is more orientated towards tibial tunnel position and retrograde screw insertion and does not concern femoral tunnel in details. Further on, femoral tunnel is drilled through the tibial tunnel making it almost impossible to achieve anatomical position of the ACL, position of the femoral subtunnels is different (01:35 for the left knee) and finally our technique describes a unique way of graft introduction that ensures that the graft stays in place and represents the natural ligament bundles. Other similar techniques use complex (AperFix System, Cayenne Medical) [14] and more expensive fixation system (Femoral INTRAFIX, DePuy Mitek). Some of them use combine BTB and hamstring tendon graft and make four tunnels to achieve double bundle [9]. Making of subtunnels and placing the two bundles of the graft in them enables forming AM and PL bundle and also, decreases pressure at the tunnel walls thus significantly reducing risk of the "blow out" effect. Further on, an advantage of this technique is the position of the femoral tunnel which in relation to the Resident's ridge mostly corresponds to the anatomical position of a natural ACL. Together with bundles separation inside the tunnel, this additionally enables achieving optimal position of the bundles so that they almost completely resemble the bundles of a natural ACL [15]. It has been proven that the weakest link in ACL reconstruction is initial graft stability [8]. Rigid fixation avoids "bungee" and "windshield wiper" effect described at suspended fixation. Finally, making suture in the middle of the graft and forming the "body" reduces the risk of graft damage and rupturing described as a complication of other rigid fixation techniques.

\section{Conclusion}

This technique is simple, it eliminates or significantly reduces risk described at other both rigid and suspended fixation techniques. The "bungee" and "windshield wiper" effects are eliminated, the anatomic position of the graft is achieved and the risk of the "blow out" effect and graft damage during screw insertion is significantly reduced. This technique allows body to renew the ACL that will in anatomic and biomechanical aspect almost completely represent the natural ACL.

\section{Conflicts of Interest}

Authors have no conflicts of interest to disclose.

\section{Bibliography}

1. Brown CH., et al. "The use of hamstring tendons for anterior cruciate ligament reconstruction: technique and results". Clinics in Sports Medicine 12 (1993): 723-756.

2. Fu FH., et al. "Current trends in anterior cruciate ligament reconstruction, part 1: biology and biomechanics of reconstruction". American Journal of Sports Medicine 27 (1999): 821-830.

3. Shultz WR and Carr CF. "Comparison of clinical outcomes of reconstruction of anterior cruciate ligament: autogenous patellar tendon and hamstring grafts". The American Journal of Orthopedics 31 (2002): 613-620.

4. Eriksson K., et al. "A comparison of quadruple semitendinosus and patellar tendon grafts in reconstruction of the anterior cruciate ligament". The Journal of Bone and Joint Surgery British 83 (2001): 348-354.

5. Rodeo SA., et al. "Tendon healing in a bone tunnel: a biomechanical and histological study in the dog". The Journal of Bone and Joint Surgery American 75 (1993): 1795-180.

6. Aune AK., et al. "Intereference screw fixation of hamstrings vs. patellar tendon grafts for anterior cruciate ligament reconstruction". Knee Surgery, Sports Traumatology, Arthroscopy 6 (1998): 99-102.

7. Clatworthy MG., et al. "Tunnel widening in anterior cruciate ligament reconstruction: a prospective evaluation of hamstring and patella tendon grafts". Knee Surgery, Sports Traumatology, Arthroscopy 7 (1999): 138-145.

8. Mologne TS and Friedman MJ. "Graft options for ACL reconstruction". The American Journal of Orthopedics 29 (2000): 845-853.

9. Dejour D., et al. "Double-bundle bone-patellar tendon-bone and gracilis in ACL reconstruction". Knee Surgery, Sports Traumatology, Arthroscopy 20 (2012): 2239-2242.

10. Beard D., et al. "Hamstrings vs. patella tendon for anterior cruciate ligament reconstruction: a randomised controlled trial". Knee 8 (2001): 45-50.

11. Purnell ML., et al. "Anterior cruciate ligament insertions on the tibia and femur and their relationships to critical bony landmarks using high-resolution volume rendering computed tomography". American Journal of Sports Medicine 36 (2008): 2083-2090. 
12. Schreiber VM., et al. "Anatomic double-bundle ACL reconstruction". Sports Medicine and Arthroscopy 18 (2010): 27-32.

13. Morgan CD., et al. "Anatomic tibial graft fixation using a retrograde bio interference screw for endoscopic anterior cruciate ligament reconstruction". Arthroscopy 18.7 (2002): e38.

14. Uribe JW., et al. "Two-year outcome with the Aper-Fix System for ACL reconstruction". Orthopedics 36 (2013): 159-164.

15. Mae T., et al. "Force sharing between two grafts in the anatomical two-bundle anterior cruciate ligament reconstruction". Knee Surgery, Sports Traumatology, Arthroscopy 14 (2006): 505-509.

\section{Assets from publication with us}

- Prompt Acknowledgement after receiving the article

- Thorough Double blinded peer review

- Rapid Publication

- Issue of Publication Certificate

- High visibility of your Published work

Website: https://www.actascientific.com/

Submit Article: https://www.actascientific.com/submission.php

Email us: editor@actascientific.com

Contact us: +919182824667 